\title{
Smart Bike: Sistema de aluguel de bicicletas
}

\author{
Thamer R. Leme, Francisco A. S. do Carmo, Guilherme P. Aquino e Evandro C. Vilas Boas
}

\begin{abstract}
Resumo - Esse trabalho apresenta o desenvolvimento de um sistema de controle de acesso e monetização para empréstimo de bicicleta, o Smart Bike. Esse sistema compreende um aplicativo móvel, banco de dados e dois módulos de hardware. Desenvolveuse o aplicativo em linguagem Java para sistema operacional Android e o banco de dados pela plataforma LAMP. Os módulos de hardware referem-se à plataforma para controle de travas da bicicleta e ao sistema de localização do veículo em movimento. A comunicação entre os sistemas ocorre por intermédio de um broker MQTT (Message Queuing Telemetry Transport).
\end{abstract}

Palavras-Chave-IoT, MQTT, sistema de aluguel de bicicletas.

Abstract- This work describes the access control and monetization system development, which allows rental bicycles, named Smart Bike. The systems comprise a mobile application, database, and two hardware modules. It uses the Java language and the Android Studio platform to develop the application. The database has been implemented through the LAMP platform. The hardware modules refer to the bicycle lock control platform and the location system. The mobile application and firmware communicate through a broker server using Message Queuing Telemetry Transport (MQTT) protocol.

Keywords - bike rental system, IoT, MQTT.

\section{INTRODUÇÃO}

$\mathrm{O}$ advento da Internet das Coisas (Internet of Things, IoT) tornou possível o desenvolvimento de aplicações e soluções práticas em diversas áreas [1]. Objetos cotidianos e sensores se conectam através de uma rede de telecomunicações ou da Internet para troca de dados. As aplicações IoT se estendem as mais diversas áreas da sociedade como a mobilidade urbana. Nesse contexto, citam-se modelos de negócios baseados em aluguel de bicicletas e/ou patinetes por meio da prática by your self [2-4]. O usuário aluga uma bicicleta em uma determinada localidade e a devolve em outra. Realizam-se as transações financeiras e liberação/devolução das bicicletas por meio de aplicativos. No Brasil, existem algumas soluções em projetos de bicicletas comunitárias como as bicicletas do Itáu, Yellow e Uber [2-4]. O modelo de negócios do Itaú utiliza plataformas distribuídas geograficamente para ancorar as bicicletas. Já o modelo de empréstimo de bicicletas da Yellow e Uber é dockless, ou seja, emprestam-se/devolvem-se as bicicletas em qualquer ponto geográfico da cidade. Essas bicicletas possuem um sistema de trava próprio e dispensam o uso de plataformas.

Com o intuito de desenvolver um sistema similar aos supracitados, iniciou-se o projeto de um sistema para controle de acesso e monetização de locação de bicicletas [5]. Esse projeto integrará o Smart Campus do Instituto Nacional de Telecomunicações (Inatel). Na primeira fase, desenvolveu-se a prova de conceito dos sistemas de identificação de usuário e liberação de uso, rastreamento de percurso e demonstração de rota ao usuário [9]. Na segunda fase do projeto, que compreende

Thamer R. Leme, Francisco A. S. do Carmo, Guilherme P. Aquino e Evandro C. V. Boas, Laboratório de Cyber Segurança e Internet das Coisas (CS\&I Lab.), Instituto Nacional de Telecomunicações (Inatel) thamer.reis@get.inatel.br, francisco.assis@inatel.br, guilhermeaquino@inatel.br, evandro.cesar@inatel.br. esse trabalho, aprimoraram-se esses sistemas e implementaramse o módulo referente a plataforma para liberação/devolução das bicicletas, aplicativo móvel para Android, banco de dados e um broker próprio. Estruturou-se o trabalho em três seções. $\mathrm{Na}$ Seção II, discute-se o desenvolvimento do projeto, explorando aspectos de software e hardware. Apresentam-se, os principais comentários, conclusões e trabalhos futuros na Seção III.

\section{SMART BIKE}

O projeto Smart Bike tem como objetivo controlar o acesso às bicicletas e automatizar a monetização durante o uso. $\mathrm{O}$ sistema integra um aplicativo móvel, um banco de dados e módulos de hardware para a bicicleta e a plataforma. Optou-se pelo modelo de plataformas para armazenar as bicicletas, assemelhando-se ao sistema da Bike Itaú. As quatro entidades se comunicam por meio de um broker MQTT, como visto na Figura 1. Tem-se três processos principais denominados de liberação, em uso e devolução. Na liberação da bicicleta, ocorre a comunicação entre a plataforma, o módulo de hardware da bicicleta e o banco de dados. Durante o uso, há comunicação entre o módulo de hardware da bicicleta, banco de dados e aplicativo móvel. Na devolução, envolvem-se as quatro entidades.

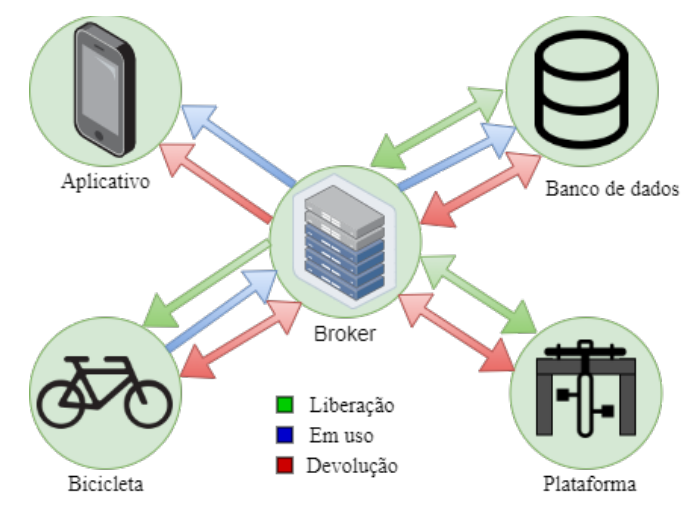

Fig. 1. Diagrama em blocos do projeto Smart Bike.

Realiza-se a liberação da bicicleta na plataforma por meio de um leitor de RFID (Radio-Frequency IDentification), que identifica a TAG da carteirinha de estudante ou crachá de colaborador. Utiliza-se a TAG para verificar se o usuário está cadastrado no sistema. Se cadastrado, a plataforma libera a bicicleta e envia um comando ao módulo da bicicleta para iniciar o processo de monetização de uso. Obtêm-se o valor cobrado por meio de uma relação entre tempo de uso e deslocamento. Caso a TAG não esteja no sistema, a plataforma notifica o usuário e solicita o cadastro. Durante o deslocamento, o módulo de hardware da bicicleta envia informações de latitude e longitude ao banco de dado, que são acessados pelo aplicativo móvel para traçar a rota atual. Na devolução, o usuário deve posicionar a bicicleta na plataforma e acionar a trava por meio da carteirinha/crachá. Dessa forma, a plataforma trava a bicicleta e envia um comando ao módulo da bicicleta para finalizar o processo de monetização, que envia as informações para o banco de dados e aplicativo móvel. 
Desenvolveu-se o aplicativo móvel utilizando o ambiente de desenvolvimento integrado Android Studio e linguagem de programação Java. $\mathrm{O}$ aplicativo permite a interação entre o usuário e o sistema. Na Figura 2, apresentam-se as telas do aplicativo. Tem-se a tela para acesso à área de usuário ou cadastro de novos usuários. A área de usuário permite acesso aos dados da corrida e rota durante um deslocamento, assim como a localização das plataformas e ao histórico de corridas.

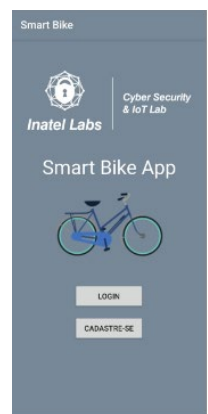

Tela de acesso/registro

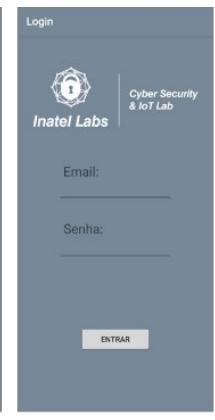

Tela de $\log$ in

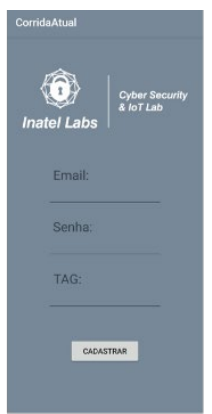

Tela de cadastro

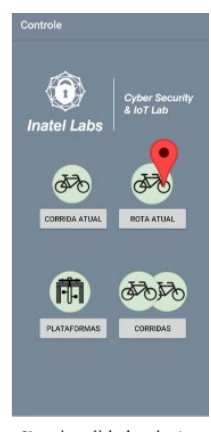

Funcionalidades do App
Fig. 2. Aplicativo móvel.

O projeto possui dois módulos de hardware: a plataforma e o hardware da bicicleta. A plataforma controla o sistema de trava da bicicleta. $\mathrm{O}$ módulo instalado na bicicleta realiza o processo de monetização e envio de localização em tempo real. Na Figura 3, mostra-se ambos os módulos com os respectivos componentes. A plataforma constitui-se de um microcontrolador ESP8266 que é responsável pelo controle do sistema de trava da bicicleta e módulo leitor RFID RC522. O ESP8266 trata o sinal captado pelo leitor de RFID para obter a TAG em formato hexadecimal. Emprega-se essa TAG para identificar o usuário nos processos explicitados na Figura 1. Durante o processo de liberação e devolução da bicicleta, o ESP8266 atua sobre o sistema de trava que integra um relé e uma trava eletromagnética. $\mathrm{O}$ microcontrolador também se comunica com as demais entidades por meio do servidor broker MQTT tanto no envio de dados quanto na recepção, tratamento e atuação.

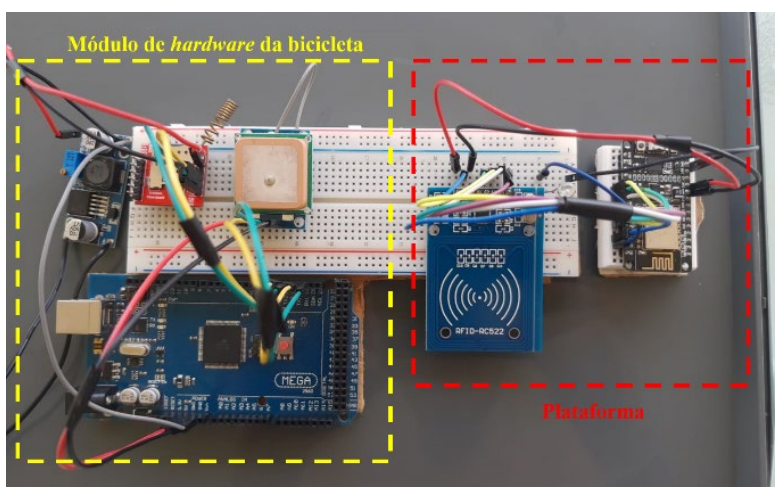

Fig. 3. Plataforma e módulo de hardware da bicicleta.

O módulo da bicicleta compreende um microcontrolador Arduíno Mega, módulo GPS (global positioning system) e módulo GPRS (general packet radio service). O módulo GPS modelo GY-NEO6MV2 é responsável por enviar informações de latitude e longitude ao microcontrolador, que trata esses dados e envia para o banco de dados e aplicativo móvel, por intermédio do broker, durante o processo de uso e devolução da bicicleta. Incluiu-se no projeto o módulo GPRS modelo Sim8001 para obter comunicação em ambientes desprovidos de conexão com a Internet via redes WiFi por meio da rede de telefonia móvel. Estruturou-se um banco de dados para armazenar informações referentes ao cadastro de usuários, monetização e demais dados relacionados ao trajeto, como visto na Figura 4. Alojou-se o banco de dados em uma máquina virtual do ambiente virtual do Laboratório de Cyber Segurança e Internet das Coisas (CS\&I Lab.). Empregou-se a plataforma de código aberto LAMP. Para o projeto, criaram-se um banco de dados para registro de usuários, registro de corridas, registro de plataformas e registro de localização.

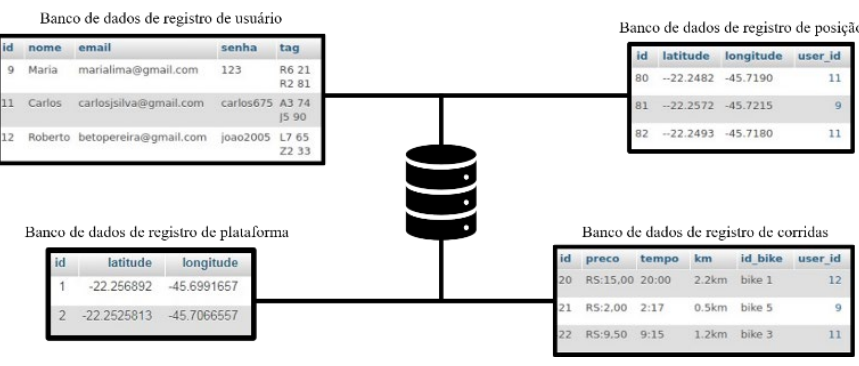

Fig. 4. Banco de dados para a Smart Bike.

\section{CONCLUSÕES}

Esse trabalho apresentou o desenvolvimento de um sistema para controle de acesso e monetização no aluguel de bicicletas para o Smart Campus do Inatel. O projeto integra um aplicativo móvel, bancos de dados e módulos de hardware para a bicicleta e a plataforma. Empregou-se um servidor broker MQTT para a comunicação entre as partes supracitadas. O projeto evoluiu consideravelmente em relação a primeira versão com o aprimoramento dos sistemas de identificação de usuário, liberação de uso, rastreamento de percurso e demonstração de rota ao usuário. Além disso, implementaram-se o sistema da plataforma para liberação/devolução das bicicletas e uma interface de interação com o usuário por meio do aplicativo móvel. Trabalhos futuros compreendem a integração do módulo de hardware em uma bicicleta e a elaboração de uma plataforma modelo. Além disso, visa-se implementar mecanismos de segurança no processo de comunicação para prover segurança no tráfego e manipulação de dados.

\section{AGRADECIMENTOS}

Os autores agradecem ao Instituto Nacional de Telecomunicações - Inatel, por prover os meios necessários a realização desse trabalho de IC.

\section{REFERÊNCIAS}

[1] Simone Cirani et al., Internet of Things: Architectures, Protocols and Standards. 1th. John Wiley Sons Ltd, 2019.

[2] Itaú, Bike Itaú. [Online] Disponível em: <https://bikeitau.com.br/ bikerio/>. Acesso: 2021-01-03.

[3] Auto Esporte. Yellow começa a oferecer bicicleta elétrica compartilhada no Brasil. [Online]. Disponível: < https://g1.globo.com/carros/noticia /2019/03/11/yellow-comeca-a-oferecer-bicicleta-eletrica-compartilhadano-brasil.ghtml>. Acesso: 2021-01-03.

[4] Aline Batista. Jump: Veja como funcionam as bicicletas elétricas da Uber. [Online]. Disponível: <https://www.techtudo.com.br/noticias/2019/09/ jump-veja-como-funcionam-as-bicicletas-eletricas-da-uber.ghtml $>$. Acesso: 2021-01-03.

[5] Y. C. T. Mendes et al., "Smart Bike Inatel," in XXXII Congresso de Iniciação Científica do Inatel - Incitel 2020, Santa Rita do Sapucaí, MG, Brasil: Instituto Nacional de Telecomunicacões, 2020, pp. 63-65. ISBN: 2359-6457. 